\title{
Targeting GATA transcription factors - a novel strategy for anti-aging interventions?
}

Andreas Zimmermann ${ }^{1}$, Katharina Kainz ${ }^{1,2}$, Sebastian J. Hofer ${ }^{1,3}$, Maria A. Bauer ${ }^{1}$, Sabrina Schroeder ${ }^{1}$ Jörn Dengjel $^{4}$, Federico Pietrocola ${ }^{5}$, Oliver Kepp ${ }^{6-9}$, Christoph Ruckenstuhl ${ }^{1}$, Tobias Eisenberg ${ }^{1,3,10,11}$, Stephan J. Sigrist ${ }^{12}$, Frank Madeo ${ }^{1,3,10, *}$, Guido Kroemer ${ }^{6-9,} 13-15, *$ and Didac Carmona-Gutierrez ${ }^{1, *}$

${ }^{1}$ Institute of Molecular Biosciences, NAWI Graz, University of Graz, Graz, Austria.

${ }^{2}$ Division of Endocrinology and Diabetology, Department of Internal Medicine, Medical University of Graz, Graz, Austria.

${ }^{3}$ BioTechMed Graz, Graz, Austria.

${ }^{4}$ Department of Biology, Université de Fribourg, Switzerland.

5 Institute for Research in Biomedicine; Barcelona, Spain

${ }^{6}$ Equipe 11 labellisée Ligue contre le Cancer, Centre de Recherche des Cordeliers, INSERM U 1138, Paris, France.

${ }^{7}$ Metabolomics and Cell Biology Platforms, Gustave Roussy Comprehensive Cancer Center, Villejuif, France.

8 8Université de Paris, Paris, France.

${ }^{9}$ Sorbonne Université, Paris, France.

${ }^{10}$ BioHealth Graz, Graz, Austria.

${ }^{11}$ Central Lab Gracia, NAWI Graz, University of Graz, Graz, Austria.

12 Institute for Biology/Genetics, Freie Universität Berlin, Berlin, Germany.

${ }^{13}$ Pôle de Biologie, Hôpital Européen Georges Pompidou, Paris, France.

${ }^{14}$ Suzhou Institute for Systems Biology, Chinese Academy of Sciences, Suzhou, China.

${ }^{15}$ Karolinska Institute, Department of Women's and Children's Health, Karolinska University Hospital, Stockholm, Sweden.

* Corresponding Author:

Frank Madeo, Institute of Molecular Biosciences, Humboldtstrasse 50/EG, 8010 Graz, Austria; E-mail: frank.madeo@uni-graz.at; Didac Carmona-Gutierrez, Institute of Molecular Biosciences, Humboldtstrasse 50/EG, 8010 Graz, Austria; E-mail: carmonag@unigraz.at;

Guido Kroemer, INSERM, U848, Institut Gustave Roussy, Pavillon de Recherche 1, 39 rue Camille Desmoulins, 94805 Villejuif, France; E-mail: kroemer@orange.fr

GATA transcription factors (TFs) constitute a conserved family of zinc-finger TFs that fulfill diverse functions across eukaryotes. Accumulating evidence suggests that GATA TFs also play a role in lifespan regulation. In a recent study, we identified a natural polyphenol, 4,4'-dimethoxychalcone (DMC), that extends lifespan depending on reduced activity of distinct GATA TFs. Prolonged lifespan by DMC treatment depends on autophagy, a protective cellular self-cleansing mechanism. In yeast, DMC reduces the activity of the GATA TF Gln3 and, genetic deletion of Gln3 is sufficient to increase autophagy levels during cellular aging. In addition, we observed similar changes in the abundance of several amino acids in the metabolome of DMCtreated and GATA/GIn3 depleted cells. Here, we examine current data on the involvement of GATA TFs in the regulation of autophagy and longevity in different organisms and explore if GATA TFs might be suitable targets for anti-aging interventions.

Global life expectancy at birth has increased over the past decades from 66.5 years in 2000 to 72.0 years in 2016: an increase of 5.5 years. In contrast, healthy life expectancy at birth, the number of years a person might live without disabilities, has only experienced an average increase of only 4.8 years between 2000 and 2016 [1]. Put simply, a

Comment on Carmona-Gutierrez D, Zimmermann A, Kainz K, Pietrocola F, Chen G, Maglioni S, Schiavi A, Nah J, Mertel S, Beuschel CB, Castoldi F, Sica V, Trausinger G, Raml R, Sommer C, Schroeder S, Hofer SJ, Bauer MA, Pendl T, Tadic J, Dammbrueck C, Hu Z, Ruckenstuhl C, Eisenberg T, Durand S, Bossut N, Aprahamian F, Abdellatif M, Sedej S, Enot DP, Wolinski H, Dengjel J, Kepp O, Magnes C, Sinner F, Pieber TR, Sadoshima J, Ventura N, Sigrist SJ, Kroemer G, Madeo F (2019). The flavonoid 4,4'-dimethoxychalcone promotes autophagy-dependent longevity across species. Nat Commun 10(1):651. doi: 10.1038/s41467-019-08555-w 
person born today will likely spend the last decade of her or his life suffering from age-associated conditions, like neurodegeneration, cardiovascular disease, diabetes or cancer. Anti-aging strategies aim at closing this gap between life- and healthspan, either by behavioral - mostly dietary - interventions or by pharmacologically targeting cellular pathways that influence aging. Among these antiaging molecules are compounds like rapamycin, which inhibits the central nutrient sensing kinase mechanistic target of rapamycin (mTOR), resveratrol, which stimulates the activity of the histone deacetylase sirtuin-1, or spermidine, which inhibits the acetyltransferase EP300 [2-5]. Thus far, dozens of anti-aging compounds have been described, and most of them act via decreased nutrient signaling and/or reduced protein acetylation, which seems to be a common hallmark among pharmacological anti-aging interventions [2]. Nevertheless, novel molecules, especially those acting via alternative pathways, are needed, since they might be used in new combinatory approaches.

In a recent study [6], we investigated different classes of flavonoids, a group of secondary metabolites from plants, for their ability to promote longevity. For that purpose, we conducted a high-throughput screen based on chronological aging of the yeast Saccharomyces cerevisiae, an established model for the aging of post-mitotic cells [7, 8]. In particular, our screen measured three different aging-associated hallmarks as readouts: (i) the loss of plasma membrane integrity, (ii) the accumulation of reactive oxygen species, and (iii) the loss of clonogenic potential. The compound that most consistently improved all three parameters was the chalcone 4,4'-dimethoxychalcone (DMC), which we detected in the Asian traditional medicine plant Angelika keiskei (also known as Ashitaba). Subsequent experiments unraveled that DMC administration prolonged lifespan in nematodes (Caenorhabditis elegans) and fruit flies (Drosophila melanogaster) and decelerated cellular senescence in human cancer cells.

Many anti-aging compounds induce autophagy, an intracellular mechanism that recycles superfluous or damaged cellular material by sequestering it in vesicles. These so-called autophagosomes subsequently fuse with lysosomes, in which the cargo is degraded to replenish building blocks for anabolic reactions [2, 9]. DMC treatment led to elevated autophagy levels in all organisms tested, including yeast, nematodes, flies, mice and cultured human cells. DMC injection protected mice from hepatotoxicity induced by acute ethanol intoxication and from cardiovascular damage induced by prolonged ischemia, which are two conditions that can be improved by autophagy induction [6]. Importantly, both lifespan extension and cardioprotection - but not liver protection - depended on intact autophagic signaling, as DMC was not able to improve these parameters upon deletion of autophagy-related (ATG) genes. Moreover - unlike many other anti-aging compounds - DMC treatment did not reduce mTOR signaling, and in yeast, the anti-aging effects depended neither on the mTOR component Tor1, nor on the sirtuin-1 homolog Sir2. Instead, a mechanistic screen in yeast revealed that
DMC required the depletion of the GATA transcription factor (TF) GIn3 to exert its anti-aging effects. DMC treatment of wildtype cells resulted in a phenotype similar to cells devoid of GIn3: both DMC treatment and GLN3 deletion reduced cell death and promoted autophagy during chronological aging. In addition, we observed similar changes in the abundance of amino acid in the metabolome of DMCtreated wildtype and $\Delta g / n 3$ cells. Importantly, DMC treatment reduced Gln3 signaling close to the levels of $\Delta g \ln 3$ cells, altogether suggesting that DMC might mimic a Gln3depleted status. GIn3 is a part of a zinc finger TF system, which also includes the GATA TFs Gat1, Gzf3 and Dal80. All these GATA family members recognize similar core consensus sequences and regulate nitrogen catabolite repression (NCR), which primarily serves to replenish amino acid biosynthesis when cells grow on poor nitrogen sources. GATA TFs can both activate and repress transcription of target genes, and all yeast GATA TFs are woven into a net of extensive cross-regulation and feedback loops [10]. Despite the functional similarity among GATA TFs, only deletion of GLN3, but not of any other GATA TF, was able to mimic the anti-aging effects of DMC [6].

GIn3 translocates to the nucleus and activates NCRresponsive genes (e.g. the ammonium permease MEP2) upon rapamycin treatment, indicating that mTOR signaling regulates $\mathrm{Gln} 3$ activity [11]. Since mTOR inhibition by rapamycin potently induces autophagy and Gln3 activation coincides with increased autophagic flux, it has long been suggested that $\mathrm{Gln} 3$ is a direct activator of genes involved in autophagy. Expression of ATG14 is reportedly induced upon deletion of Ure2, a cytosolic protein that sequesters and inhibits both GIn3 and Gat1 [12], although a more recent study has found no direct effect of GLN3 deletion on ATG14 transcription [13]. In chronological aging experiments, we observed that deletion of GLN3 resulted in increased autophagic flux, and rapamycin treatment was even more effective (both when monitoring survival and autophagy) in $\Delta g / n 3$ cells, suggesting that rapamycininduced autophagy induction and nuclear Gln3 localization are rather correlative than causal events. Importantly, GIn3 might have a different role upon acute autophagy induction, e.g. by nitrogen starvation. Indeed, 'basal' Gln3 activity seems to repress transcription of several ATG genes in rich media, while some ATG genes showed reduced transcription upon nitrogen starvation when $G \ln 3$ was absent [13]. In any case, a model where basal Gln3 activity represses autophagy during chronological aging is more consistent with the reported longevity phenotype of $\Delta g \ln 3$ cells $[6,14]$. Since $\mathrm{G} \ln 3$ regulates the expression of genes which mediate amino acid biosynthesis, it is possible that GIn3 inhibition leads to depletion of amino acids, which in turn activates autophagic flux as an alternative pathway for replenishing amino acid pools. This hypothesis is corroborated by the metabolic signature of $\Delta g \ln 3$ cells, albeit the causal mechanistic relationship between these changes and autophagy induction remains to be determined [6].

Importantly, our study corroborated that GATA TFs are involved in the beneficial effects of DMC in higher eukary- 
otes. Indeed, DMC failed to increase lifespan in nematodes with concomitant depletion of the Gln3 homolog elt-1. In addition, RNAi-mediated knockdown of elt-1 led to increased autophagosome formation, and DMC did not further increase the number of autophagosomes in elt-1 RNAi-treated animals [6]. Similarly, DMC was unable to boost autophagosome formation in human cells when GATA-2 (and to a lesser extent GATA-3 and GATA-4), but not other GATA TFs were depleted.

In multicellular organisms, GATA TFs have mainly been implicated in development, particular hematopoiesis and cardiac differentiation $[15,16]$. Interestingly, the crosstalk among different GATA TFs seems to be conserved between yeast and humans, as GATA TF switching and feedback inhibition represent important developmental control circuits in both species [17]. Nevertheless, in multicellular organisms, it is poorly investigated whether GATA TFs are involved in the regulation of amino acid biosynthesis and autophagy and whether they are influenced by mTOR signaling. In hematopoietic cells, GATA-1 overexpression activates the expression of genes involved in autophagy, although some ATGs (e.g. ATG5) are repressed [18]. In other tissues, GATA-4 accumulates with age and might contribute to the senescence-associated secretory phenotype and age-associated tissue inflammation, making it an attractive target for anti-aging interventions. Intriguingly, GATA-4 seems to be targeted by SQSTM1/p62 for autophagic degradation [19]. It is unknown, however, if GATA-4 itself can regulate autophagy. On the other hand, in nematodes, aging is accompanied by decreasing levels of the GATA TF elt-2, and accordingly, elt-2 overexpression extends lifespan [20]. In flies, the GATA TF serpent (srp), the closest Drosophila homolog of $\mathrm{Gln} 3$ has been suggested to play a role in autophagy execution, as srp knockdown prevents fusion of lysosomes with autophagosomes [21]. In contrast, a more recent study has implicated srp as a negative regulator of dietary restriction-mediated lifespan extension in flies for at least two reasons. First, genes that were differentially expressed upon restriction of essential amino acids (EAA), a condition that extends longevity, were enriched for GATA TF binding sites. Second, fatbody-specific knockdown of srp was sufficient to increase lifespan and abolished the positive effects of EAA restriction. Of note, the signature of EAA-enriched GATA-regulated genes was also observed upon rapamycin treatment, suggesting that in Drosophila, GATA TF signaling might be regulated by mTOR. Interestingly, whole-body srp knockdown had a similar effect on lifespan as dietary restriction, but reduced fecundity, suggesting that systemic depletion of this TF comes at a cost [22]. Our data yielded a similar picture: systemic elt-1 depletion in nematodes failed to increase lifespan, yet abolished the anti-aging effect of DMC [6]. As a possibility, in multicellular organisms, tissue-specific depletion of GATA TFs might yield life- or healthspan effects that cannot be seen upon whole-body depletion, but this needs to be further investigated.

Apparently, longevity-extending effects of DMC rely on an increase in autophagic flux. Still, several questions re- garding the mode of action of DMC remain to be answered:

(i) how does DMC inhibit Gln3? We could not demonstrate any physical interaction of the compound with the Gln3 protein. In fact, DMC might target Gln3 indirectly by acting on Gln3 regulators. Besides mTOR-mediated regulation, Gln3 activity can be modulated by PP2A-like protein phosphatases, although in many cases, mTOR activity inversely correlates with PP2A activity [23]. Intriguingly, DMC-mediated cytoprotection was lost in yeast strains lacking the PP2A subunits Tpd3 or Pph21/Pph22 [6]. Possible direct effects of DMC on such PP2A-like enzymes should be studied in the future.

(ii) Does DMC reduce GATA signaling in multicellular organisms? While we did not measure GATA activity in multicellular models, two pieces of information suggest that this could be the case. First, we found metabolomic Iterations in the liver and heart tissues from DMC-treated mice, particularly in amino acid-related metabolism, that are reminiscent of those found in yeast. Second, as in yeast, the pro-autophagic DMC effects are dependent on GATA TFs in nematodes and human cell culture. However, it remains to be formally demonstrated that these changes correlate with reduced GATA signaling.

(iii) Are there other factors involved in the proautophagic activity of DMC? Although our data indicate that DMC promotes autophagy in an mTOR-independent manner and requires GATA signaling, we cannot exclude further mechanistic determinants. For instance, the activity of other TFs might be required for, or influenced by GATA TFs in the context of aging. The likely complexity of such a GATA-connected transcriptional network will be a matter of further investigation. In fact, in mammals, the transcriptional regulation of autophagy alone encompasses a set of at least 20 TFs, including FOXO1/3, ATF4 and TFEB [24]. So far, except for GATA-1, GATA TFs have not been part of the transcriptional landscape of autophagy regulation. Again, future studies will have to explore to what extent GATA TFs are involved in the transcriptional control of autophagy, likely in a cell type-dependent fashion given the tissuespecific expression profile of GATA TFs in mammals [25] and the dependency of caloric restriction on GATA signaling in Drosophila [22].

GATA TF signaling has diverse functions across different phyla. It is not unlikely that an ancient system of interdependent paralogous TFs has evolved to fulfil different needs in distinc species, ranging from tight regulation of nitrogen sensing in yeast to developmental switches in multicellular organisms. Nonetheless, emerging evidence suggests that more regulatory functions are conserved across species than previously expected, although tissuespecific effects of GATA TF modulation have to be taken into account. Arguably, this complicates pharmacological anti-aging interventions, as compounds will either have to modulate GATA TF activity in a localized manner or specifically target GATA TF subtypes. Despite these constraints, accumulating data place GATA TFs in the limelight as actionable targets for postponing age-associated disease. 


\section{ACKNOWLEDGEMENTS}

F.M. and D.C-G. are grateful to the Austrian Science Fund FWF (SFB LIPOTOX F3007 \& F3012, W1226, P29203, $\mathrm{P} 29262$ ), in particular for the project P27893 ("Proautophagic polyphenols and polyamines for longevity") and the Austrian Federal Ministry of Education, Science and Research and the University of Graz for grants "Unkonventionelle Forschung" and "flysleep" (BMWFW-80.109/0001$W F / V / 3 b / 2015)$ as well as the field of excellence program BioHealth. K.K. is a fellow of the Doctoral College "Metabolic and Cardiovascular Disease" (FWFW1226) and was funded by the University of Graz. We acknowledge support from NAWI Graz and the BioTechMed-Graz flagship project "EPIAge". GK is supported by the Ligue contre le Cancer (équipe labellisée); Agence National de la Recherche (ANR) - Projets blancs; ANR under the frame of E-Rare-2, the ERA-Net for Research on Rare Diseases; Association pour la recherche sur le cancer (ARC); Cancéropôle Ile-de-France; Chancelerie des universités de Paris (Legs Poix), Fondation pour la Recherche Médicale (FRM); a donation by Elior; European Research Area Network on Cardiovascular Diseases (ERA-CVD, MINOTAUR); Gustave Roussy Odyssea, the European Union Horizon 2020 Project Oncobiome; Fondation Carrefour; High-end Foreign Expert Program in China (GDW20171100085), Institut National du Cancer (INCa); Inserm (HTE); Institut Universitaire de France; LeDucq Foundation; the LabEx Immuno-Oncology; the RHU

\section{REFERENCES}

1. GHO | By category | Life expectancy and Healthy life expectancy Data by WHO region. WHO. Available at http://apps.who.int/gho/data/view.main.SDG2016LEXREGv?lang=en [Accessed 04/16/2019].

2. Madeo F, Carmona-Gutierrez D, Hofer SJ, and Kroemer G (2019). Caloric Restriction Mimetics against Age-Associated Disease: Targets, Mechanisms, and Therapeutic Potential. Cell Metab 29(3): 592-610. doi: 10.1016/j.cmet.2019.01.018

3. Pietrocola $F$, Lachkar S, Enot DP, Niso-Santano M, Bravo-San Pedro JM, Sica V, Izzo V, Maiuri MC, Madeo F, Mariño G, and Kroemer G (2015). Spermidine induces autophagy by inhibiting the acetyltransferase EP300. Cell Death Differ 22(3): 509-516. doi: 10.1038/cdd.2014.215

4. Eisenberg T et al. (2016). Cardioprotection and lifespan extension by the natural polyamine spermidine. Nat Med 22(12): 1428-1438. doi: $10.1038 / \mathrm{nm} .4222$

5. Eisenberg $T$, Knauer $H$, Schauer A, Büttner S, Ruckenstuhl C, Carmona-Gutierrez D, Ring J, Schroeder S, Magnes C, Antonacci L, Fussi H, Deszcz L, Hartl R, Schraml E, Criollo A, Megalou E, Weiskopf D, Laun P, Heeren G, Breitenbach M, Grubeck-Loebenstein B, Herker E, Fahrenkrog B, Fröhlich K-U, Sinner F, Tavernarakis N, Minois N, Kroemer $G$, and Madeo $F$ (2009). Induction of autophagy by spermidine promotes longevity. Nat Cell Biol 11(11): 1305-1314. doi: 10.1038/ncb1975

6. Carmona-Gutierrez D et al. (2019). The flavonoid 4,4'dimethoxychalcone promotes autophagy-dependent longevity across species. Nat Commun 10(1): 651. doi: 10.1038/s41467-019-08555-w
Torino Lumière; the Seerave Foundation; the SIRIC Stratified Oncology Cell DNA Repair and Tumor Immune Elimination (SOCRATE); and the SIRIC Cancer Research and Personalized Medicine (CARPEM).

\section{CONFLICT OF INTEREST}

O.K., G.K., D.C-G. and F.M. are the scientific co-founders of Samsara Therapeutics.

\section{COPYRIGHT}

(C) 2019 Zimmermann et al. This is an open-access article released under the terms of the Creative Commons Attribution (CC BY) license, which allows the unrestricted use, distribution, and reproduction in any medium, provided the original author and source are acknowledged.

Please cite this article as: Andreas Zimmermann, Katharina Kainz, Sebastian Hofer, Maria A. Bauer, Sabrina Schroeder, Jörn Dengjel, Federico Pietrocola, Oliver Kepp, Christoph Ruckenstuhl, Tobias Eisenberg, Stephan J. Sigrist, Frank Madeo, Didac CarmonaGutierrez and Guido Kroemer (2019). Targeting GATA transcription factors - a novel strategy for anti-aging interventions? Microbial Cell 6(5): 212-216. doi: 10.15698/mic2019.05.676

7. Longo VD, and Fabrizio $P$ (2012). Chronological Aging in Saccharomyces cerevisiae. Subcell Biochem 57: 101-121. doi: 10.1007/978-94007-2561-4_5

8. Zimmermann A, Hofer S, Pendl T, Kainz K, Madeo F, and CarmonaGutierrez D (2018). Yeast as a tool to identify anti-aging compounds. FEMS Yeast Res 18(6). doi: 10.1093/femsyr/foy020

9. Yin Z, Pascual C, and Klionsky DJ (2016). Autophagy: machinery and regulation. Microbial Cell 3(12): 588-596. doi: 10.15698/mic2016.12.546

10. Ljungdahl PO, and Daignan-Fornier B (2012). Regulation of Amino Acid, Nucleotide, and Phosphate Metabolism in Saccharomyces cere visiae. Genetics 190(3): 885-929. doi: 10.1534/genetics.111.133306

11. Tate JJ, Georis I, Feller A, Dubois E, and Cooper TG (2009). Rapamycin-induced GIn3 Dephosphorylation Is Insufficient for Nuclear Localization: Sit4 AND PP2A PHOSPHATASES ARE REGULATED AND FUNCTION DIFFERENTLY. J Biol Chem 284(4): 2522-2534. doi: 10.1074/jbc.M806162200

12. Chan T-F, Bertram PG, Ai W, and Zheng XFS (2001). Regulation of APG14 Expression by the GATA-type Transcription Factor Gln3p. J Biol Chem 276(9): 6463-6467. doi: 10.1074/jbc.M008162200

13. Bernard A, Jin M, Xu Z, and Klionsky DJ (2015). A large-scale analysis of autophagy-related gene expression identifies new regulators of autophagy. Autophagy 11(11): 2114-2122. doi 10.1080/15548627.2015.1099796

14. Powers RW (2006). Extension of chronological life span in yeast by decreased TOR pathway signaling. Genes Dev 20(2): 174-184. doi: 10.1101/gad.1381406 
15. Cohen ML, Kim S, Morita K, Kim SH, and Han M (2015). The GATA Factor elt-1 Regulates C. elegans Developmental Timing by Promoting Expression of the let-7 Family MicroRNAs. PLOS Genet 11(3): e1005099. doi: 10.1371/journal.pgen.1005099

16. Tremblay M, Sanchez-Ferras O, and Bouchard M (2018). GATA transcription factors in development and disease. Development 145(20): dev164384. doi: 10.1242/dev.164384

17. Bresnick EH, Lee $H-Y$, Fujiwara $T$, Johnson KD, and Keles S (2010). GATA Switches as Developmental Drivers. J Biol Chem 285(41): 31087-31093. doi: 10.1074/jbc.R110.159079

18. Kang Y-A, Sanalkumar R, O'Geen H, Linnemann AK, Chang C-J, Bouhassira EE, Farnham PJ, Keles S, and Bresnick EH (2012). Autophagy Driven by a Master Regulator of Hematopoiesis. Mol Cell Biol 32(1): 226-239. doi: 10.1128/MCB.06166-11

19. Kang C, Xu Q, Martin TD, Li MZ, Demaria M, Aron L, Lu T, Yankner BA, Campisi J, and Elledge SJ (2015). The DNA damage response induces inflammation and senescence by inhibiting autophagy of GATA4 Science 349(6255): aaa5612-aaa5612. doi: 10.1126/science.aaa5612

20. Mann FG, Van Nostrand EL, Friedland AE, Liu X, and Kim SK (2016). Deactivation of the GATA Transcription Factor ELT-2 Is a Major Driver of Normal Aging in C. elegans. PLOS Genet 12(4): e1005956. doi: 10.1371/journal.pgen.1005956
21. Bánréti Á, Lukácsovich T, Csikós G, Erdélyi M, and Sass M (2012). PP2A regulates autophagy in two alternative ways in Drosophila. Autophagy 8(4): 623-636. doi: 10.4161/auto.19081

22. Dobson AJ, He X, Blanc E, Bolukbasi E, Feseha Y, Yang M, and Piper MDW (2018). Tissue-specific transcriptome profiling of Drosophila reveals roles for GATA transcription factors in longevity by dietary restriction. Npj Aging Mech Dis 4(1). doi: 10.1038/s41514-018-0024-4

23. Tate JJ, Georis I, Dubois E, and Cooper TG (2010). Distinct Phosphatase Requirements and GATA Factor Responses to Nitrogen Catabolite Repression and Rapamycin Treatment in Saccharomyces cerevisiae. J Biol Chem 285(23): 17880-17895. doi: 10.1074/jbc.M109.085712

24. Feng Y, Yao Z, and Klionsky DJ (2015). How to control selfdigestion: transcriptional, post-transcriptional, and post-translational regulation of autophagy. Trends Cell Biol 25(6): 354-363. doi: 10.1016/j.tcb.2015.02.002

25. Lentjes $M H$, Niessen $H E$, Akiyama $Y$, de Bruïne AP, Melotte $V$, and van Engeland $M$ (2016). The emerging role of GATA transcription factors in development and disease. Expert Rev Mol Med 18. doi: 10.1017/erm.2016.2 